\title{
High-sensitivity C-reactive protein as a marker of cardiovascular risk in obese children and adolescents
}

\author{
Hatem Hamed El-shorbagy ${ }^{1^{\star}}$, Ibraheim Abdel-aziz Ghoname ${ }^{2}$ \\ ${ }^{1}$ Departments of Pediatrics, Faculty of Medicine, Menoufia University and Clinical Pathology, Shibin el Kom, Egypt \\ ${ }^{2}$ Departments of Pediatrics, Faculty of Medicine Al-azhar University, Cairo, Egypt; *Corresponding Author: hatem732000@yahoo.com
}

Received 20 February 2010; revised 19 April 2010; accepted 20 April 2010.

\begin{abstract}
Background and aim of the work: High-sensitivity C-reactive protein (hsCRP) is a marker of low grade inflammatory state, which characterises an atherosclerotic process. The metabolic syndrome is associated with insulin resistance and a systemic low-grade inflammatory state. These disorders may arise at a very early age in obese children. We aimed to assess the utility of (hsCRP) as a marker of cardiovascular risk in obese children and adolescents. Patients and methods: This study was conducted on 100 obese child and adolescents (6-16 years). 50 apparently healthy children of matched age and sex served as control. All patients and controls were subjected to: 1-complete history taking. 2-anthropometric measurements and clinical examination including body height, weight, waist circumference, body mass index and blood pressure. 3-laboratory investigations including fasting glucose, lipid profile, apolipoprotiens and (hsCRP) were assessed. Metabolic syndrome patients had to meet three out of five criteria: concentration of triglycerides (TG) $\geq 110$ $\mathrm{mg} / \mathrm{dL}$, high density lipoprotein cholesterol (HDLC) $\leq \mathbf{4 0} \mathrm{mg} / \mathrm{dL}$, waist circumference $\geq$ 90th percentile, glucose concentration $\geq 110 \mathrm{mg} / \mathrm{dL}$, and systolic or diastolic blood pressure $\geq 90$ th percentile. Results, height, weight BMI and blood pressure were significantly higher in the obese than the control. Obese group had significantly higher (hsCRP) levels than control group, ( $p<$ 0.01 ) and significantly higher LDL-C, triglyceride (TG), and lower HDL-C than the control group. Log (hsCRP) showed a positive correlation with BMI $(p<0.001)$, blood pressure, and TG. The prevalence of the metabolic syndrome was $24 \%$.

Aim of the work: We aimed to assess the utility of the high-sensitivity $\mathrm{C}$-reactive protein (hsCRP) as a marker of cardiovascular risk in obese children and adolescents.
\end{abstract}

Mean concentrations of (hsCRP) were higher among patients who had the metabolic syndrome. Among whom, $35 \%$ had a concentration of (hsCRP) $>3.0 \mathrm{mg} / \mathrm{L}$, a concentration considered to place adults at high risk for cardiovascular disease. In multiple logistic regression analysis only abdominal obesity was significantly associated with (hsCRP). Conclusion: metabolic syndrome and abdominal obesity among our patients predispose to cardiovascular disease later in life through early low grade inflammation. (hsCRP) is one of the inflammatory markers that can be easily estimated in these patients.

Keywords: HsCRP; Cardiovascular Risk; Obesity

\section{INTRODUCTION}

The prevalence of childhood obesity has more than doubled in the last 15 years in many regions of the world [1]. This phenomenon is associated with a rapidly increasing trend in cases of type 2 diabetes in childhood. Obesity in childhood also seems to harbor a number of risk factors for cardiovascular disease (CVD) in adult life, but is not yet clear whether these are determined by glycemia, degree of obesity, or other demographic, clinical, or biochemical features of the obese child [2]. Childhood obesity seems to contribute to the development of vascular inflammation and the progression of arterial wall changes. (hsCRP) has recently emerged as a useful biomarker for vascular inflammation associated with atheRosclerosis [3]. Of novel risk factors for cardiovascular disease currently under investigation, high-sensitivity $\mathrm{C}$-reactive protein (hsCRP) is the most promising. To date, more than 20 prospective epidemiologic studies have demonstrated that (hsCRP) independently predicts vascular risk [4]. CRP is a major inflammatory cytokine that functions as a nonspecific defense mechanism in 
response to tissue injury or infection. Synthesized mainly in the liver, CRP activity is stimulated by other cytokines, especially interleukin (IL)-6, IL-1 $\beta$, and tumor necrosis factor- $\alpha$ (TNF- $\alpha)$. Accumulating evidence suggests that CRP, which is also found within macrophages of atheromatous plaques, is causally or mechanistically related to atherothrombosis [5]. The metabolic syndrome has generated a great deal of interest in recent years. Comprised of a constellation of anthropometric, physiologic, and biochemical abnormalities, the metabolic syndrome is a risk factor for cardiovascular disease and diabetes among adults. However, research about the metabolic syndrome among children and adolescents and the implications of having the metabolic syndrome is limited [6]. The metabolic syndrome has been defined as a cluster of risk factors for atherosclerotic cardiovascular disease that includes insulin resistance, dyslipidemia, abdominal adiposity, and often hypertension [7].

\section{PATIENTS AND METHODS}

This study was conducted on 100 obese child and adolescent (simple obesity) their ages ranged from (6-16 years) enrolled from pediatric outpatient clinic, Prince Sultan Armed Forces Hospital, Saudia Arabia. 50 apparently healthy children of matched age and sex served as control.

\section{Exclusion Criteria:}

1) Smokers.

2) Under any regular medication.

3) Family history of premature vascular disease.

4) Any symptoms of infection during the 2 weeks before the study.

5) Underlying etiology (secondary obesity).

All patients and controls were subjected to:

1) Complete history taking

2) Anthropometric measurement and clinical examination: All anthropometric measurements were taken with stress on body height and weight were measured in light clothes using a portable standiometer. Body mass index (BMI) was calculated as weight divided by the square of the height $\left(\mathrm{kg} / \mathrm{m}^{2}\right)$. In children, $>95$ th percentile for BMI growth chart is considered obese (4), so we used BMI $>30$ as our prospectively defined criterion for obesity, and BMI $<25$ was defined as the non-obese control level [8].

Waist circumference was measured at the high point of the iliac crest to the nearest $0.1 \mathrm{~cm}$ at the end of normal expiration with a steel measuring tape. Blood pressure measurements were obtained for each participant in the study. Participants were seated with their right arm resting at the level of the heart. Blood pressure was measured with a mercury-gravity sphygmomanometer. Child, adult, and large arm-cuff sizes were available.All measures were evaluated according to appropriate centiles. Characteristics of studied groups was shown in Table 1, There was found that height, weight and BMI were significantly higher in the obesity group than in the control group. BMI was $27.20 \pm 12.30 \mathrm{~kg} / \mathrm{m}^{2}$ in the obesity group and was $16.68 \pm 2.00 \mathrm{~kg} / \mathrm{m}^{2}$ in the control group. Obese children had significantly higher systolic blood pressure (SBP) $(115.0 \pm 9.95 \mathrm{mmHg}$ vs. $95.0 \pm 7.82$ $\mathrm{mmHg}$ ) and diastolic blood pressure (DBP) (75.85 \pm $5.03 \mathrm{mmHg}$ vs. $68.28 \pm 7.45 \mathrm{mmHg}$ ).

\section{LABORATORY INVESTIGATIONS}

Fasting glucose: concentration was measured using the glucose hexokinase method [9].

Serum lipids and apolipoprotein: Venous blood samples were taken in the morning after overnight fasting (10-12 hr). Serum total cholesterol (TC), low density lipoprotein cholesterol (LDL-C), high density lipoprotein cholesterol (HDL-C), and triglyceride (TG) concentrations were measured by standard enzymatic methods with the use of Boehringer Mannheim GmbH and a fully automatic analyzer. Serum apolipoprotein A-1 (Apo A-1), apolipoprotein B (Apo B), and apolipoprotein E (Apo E) were measured by immunonephelometry (Behring $\mathrm{Ne}$ phelometer II, Dade Behring, Inc., Newark, DE [10].

High-sensitivity C-reactive protein was assessed with the immunonephelometric assay method using company reagents (assessments made using high sensitivity method with a latex intensification). High-sensitivity C-reactive protein A BN II Nephelometer Analyzer (Dade Behring, Inc.) was used to measure serum (hsCRP) values using a high sensitivity latex-enhanced immunonephelometric assay method. The detection limit of this assay was 0.17 $\mathrm{mg} / \mathrm{dL}$. Because the 90th percentile of normal CRP distribution is $0.3 \mathrm{mg} / \mathrm{dL}$, we included only patients with

Table 1. Comparison of characteristics between obesity and control groups.

\begin{tabular}{cccc}
\hline & Obesity $(\mathrm{N}=100)$ & Control $(\mathrm{N}=50)$ & $\mathrm{P}$ \\
\hline Age & $11.82 \pm 0.6$ & $11 \pm 0.2$ & NS \\
Height & $134.23 \pm 4.0$ & $125.13 \pm 6.0$ & $<0.01$ \\
Weight & $68.05 \pm 14.40$ & $31.56 \pm 7.28$ & $<0.01$ \\
BMI & $27.20 \pm 12.30$ & $16.68 \pm 2.00$ & $<0.01$ \\
Systolic BP & $115.0 \pm 9.95$ & $95.0 \pm 7.82$ & $<0.01$ \\
Diastolic BP & $75.85 \pm 5.03$ & $68.28 \pm 7.45$ & $<0.01$ \\
\hline
\end{tabular}

NS: not significant 
CRP values below $0.3 \mathrm{mg} / \mathrm{dL}$ to avoid the influence of acute infection [11]. Metabolic syndrome, Patients had to meet three out five criteria: concentration of triglycerides $\geq 110 \mathrm{mg} / \mathrm{dL}$, HDL cholesterol $\leq 40 \mathrm{mg} / \mathrm{dL}$, waist circumference $\geq 90$ th percentile (sex specific), glucose concentration $\geq 110 \mathrm{mg} / \mathrm{dL}$, and systolic or diastolic blood pressure $\geq 90$ th percentile (age, height, and sex specific) [6].

Statistical analysis: Data was collected and expressed in tables. SPSS version computer program was used for all statistical calculations. Results were expressed as mean \pm standard deviation. Geometric mean and standard error were calculated. Comparisons between two groups were performed by the independent student-t test. Univariate and multivariate regression analyses were used to delineate the relationships between components of metabolic syndrome and (hsCRP). Due to the skewed distribution of (hsCRP) levels, (hsCRP) values were logarithmically transformed prior to regression analyses. For all analyses, probability (p) values below 0.05 were considered statistically significant tests were performed to compare mean and log-transformed concentrations of hsCRP. We examined the independent contribution of the five components of the metabolisc syndrome to (hsCRP) concentration in multiple linear regression analyses. For regression analyses, (hsCRP) concentration was log transformed to improve the distribution of this variable [12].

\section{RESULTS}

Results of our study were expressed in the following tables:

Table 2 shows comparison of metabolic parameters between obesity and control groups: Obese group had significantly higher hs-CRP levels than control group, hs-CRP levels were $1.40 \pm 0.78 \mathrm{mg} / \mathrm{dL}$ vs. $0.56 \pm 0.47$. $\mathrm{mg} / \mathrm{dL}, \mathrm{p}<0.01$ and significantly higher LDL-C, TG, Apo B and lower HDLC than the control group. TC and Apo E were higher and Apo A-1 was lower in the obesity group than in the control group, but these differences were statistically insignificant.

Table 3 shows Correlation of log hs-CRP with BMI, BP, lipid profile, and apolipoprotein concentrations: Log [hsCRP] showed a positive correlation with BMI $(\mathrm{r}=$ $0.464, \mathrm{p}<0.001)$, SBP $(\mathrm{r}=0.207, \mathrm{p}<0.05)$, DBP $(\mathrm{r}=$ $0.225, \mathrm{p}<0.05)$, Apo E $(\mathrm{r}=0.272, \mathrm{p}<0.01)$, and TG $(\mathrm{r}$ $=0.298, \mathrm{p}<0.05)$ by simple regression.

Table 4 shows Unadjusted mean concentrations \pm SE, geometric mean concentrations $\pm \mathrm{SE}$, and percentage \pm $\mathrm{SE}$ of CRP $>3.0 \mathrm{mg} / \mathrm{L}$ by presence or absence of metabolic syndrome or its five components among patients:
Among all patients $50 \%$ had no components of metabolic syndrome, $20 \%$ had one component, $6 \%$ had two components, $20 \%$ had three components, and $4 \%$ had four components. No participants had all five components. The prevalence of the metabolic syndrome was $24 \%$ (no sex difference was found, P $>0.05$ ). Furthermore, $40 \%$ had a large waist circumference, $32 \%$ had

Table 2. Comparison of metabolic parameters between obesity and control groups.

\begin{tabular}{cccc}
\hline $\begin{array}{c}\text { metabolic } \\
\text { parameters }\end{array}$ & Obesity $(\mathrm{N}=100)$ & Control $(\mathrm{N}=50)$ & $\mathrm{P}$ \\
\hline $\mathrm{TC}(\mathrm{mg} / \mathrm{dL})$ & $180.45 \pm 25.30$ & $160.23 \pm 24.67$ & $\mathrm{NS}$ \\
$\begin{array}{c}\mathrm{HDL}-\mathrm{C} \\
(\mathrm{mg} / \mathrm{dL})\end{array}$ & $53.40 \pm 11.78$ & $61.45 \pm 14.08$ & $<0.05$ \\
$\begin{array}{c}\mathrm{LDL}-\mathrm{C} \\
(\mathrm{mg} / \mathrm{dL})\end{array}$ & $105.04 \pm 22.70$ & $92.62 \pm 22.61$ & $<0.05$ \\
$\mathrm{TG}(\mathrm{mg} / \mathrm{dL})$ & $130.45 \pm 68.37$ & $88.23 \pm 45.23$ & $<0.01$ \\
$\begin{array}{c}\mathrm{Apo} \mathrm{A} 1 \\
(\mathrm{mg} / \mathrm{dL})\end{array}$ & $65.13 \pm 21.67$ & $68.65 \pm 22.55$ & $\mathrm{NS}$ \\
$\begin{array}{c}\mathrm{Apo} \mathrm{B} \\
(\mathrm{mg} / \mathrm{dL})\end{array}$ & $27.78 \pm 30.0$ & $20.00 \pm 8.47$ & $<0.05$ \\
$\begin{array}{c}\text { Apo E } \\
(\mathrm{mg} / \mathrm{dL})\end{array}$ & $2.89 \pm 1.23$ & $2.32 \pm 1.35$ & $\mathrm{NS}$ \\
$\begin{array}{c}\mathrm{hs}-\mathrm{CRP} \\
(\mathrm{mg} / \mathrm{L})\end{array}$ & $1.40 \pm 0.78$ & $0.56 \pm 0.47$ & $<0.01$ \\
\hline
\end{tabular}

TC, total cholesterol; HDL-C, high density lipoprotein cholesterol; LDL-C, low density lipoprotein cholesterol; TG, triglyceride; Apo A-1, apolipoprotein A-1; Apo B, apolipoprotein B; Apo E, apolipoprotein E; NS, not significant; $p$, p-value.

Table 3. Correlation of $\log$ (hsCRP) with BMI, BP, lipid profile, and apolipoprotein concentrations.

\begin{tabular}{ccc}
\hline Variables & hs-CRP R & $\mathrm{P}$ \\
\hline BMI & 0.464 & $<0.001$ \\
SBP & 0.207 & $<0.05$ \\
DBP & 0.225 & $<0.05$ \\
TC & 0.087 & NS \\
LDL & 0.101 & NS \\
HDL & 0.113 & NS \\
TG & 0.298 & $<0.01$ \\
ApoA & 0.173 & NS \\
ApoB & 0.015 & NS \\
ApoE & 0.272 & $<0.01$ \\
\hline
\end{tabular}

BMI, body mass index; SBP, systolic blood pressure; DBP, diastolic blood pressure; PP, pulse pressure; TC, total cholesterol; LDL-C, low density lipoprotein cholesterol; HDL-C, high density lipoprotein cholesterol; TG, triglyceride; Apo A-1, apolipoprotein A-1; Apo B, apolipoprotein B; Apo E, apolipoprotein E; r, correlation coefficient; p, p-value. 
Table 4. Unadjusted mean concentrations $\pm \mathrm{SE}$, geometric mean concentrations $\pm \mathrm{SE}$, and percentage $\pm \mathrm{SE}$ of $\mathrm{CRP}>3.0 \mathrm{mg} / \mathrm{L}$ by presence or absence of metabolic syndrome or its five components among patients.

\begin{tabular}{|c|c|c|c|c|c|c|c|c|c|c|c|}
\hline $\begin{array}{l}\text { Metabolic syndrome } \\
\text { or components }\end{array}$ & $\begin{array}{l}\text { Sample } \\
\text { Yes }\end{array}$ & Size No & Mean Yes & + _SE No & $(\mathrm{mg} / \mathrm{l}) \mathrm{P}$ & $\begin{array}{l}\text { Geometric } \\
\text { Yes }\end{array}$ & $\begin{array}{c}\text { mean +_SE } \\
(\mathrm{mg} / \mathrm{l}) \\
\text { No }\end{array}$ & $\mathrm{p}$ & $\underset{\text { Yes }}{\% \text { SE of }}$ & CRP No & $>3 \mathrm{mg} / \mathrm{p}$ \\
\hline Metabolic syndrome & 25 & 75 & $3.8 \pm 1.2$ & $1.3 \pm 0.2$ & $<0.05$ & $1.9 \pm 0.5$ & $0.4 \pm 0.04$ & $<0.001$ & & $12.7 \pm 0.3$ & $<0.05$ \\
\hline Abdominal obesity & 40 & 60 & $3.8 \pm 0.3$ & $0.9 \pm 0.4$ & $<0.001$ & $1.8 \pm 0.4$ & $0.5 \pm 0.02$ & $<0.001$ & $33.0 \pm 5.0$ & $5.4 \pm 0.8$ & $<0.001$ \\
\hline Hypertriglyceridemia & 32 & 68 & $1.6 \pm 0.4$ & $1.4 \pm 0.8$ & $>0.05$ & $0.6 \pm 0.2$ & $0.4 \pm 0.04$ & $<0.05$ & $12.6 \pm 2.1$ & $10.8 \pm 109$ & $>0.05$ \\
\hline Low HDL cholesterol & 28 & 72 & $2.1 \pm 0.9$ & $1.6 \pm 0.6$ & $>0.05$ & $0.8 \pm 0.3$ & $0.4 \pm 0.03$ & $<0.05$ & $12.4 \pm 0.3$ & $8.8 \pm 0.6$ & $>0.05$ \\
\hline High blood pressure & 18 & 82 & $5.1 \pm 0.3$ & $1.7 \pm 0.8$ & $>0.05$ & $0.7 \pm 0.4$ & $0.5 \pm 0.07$ & $<0.05$ & $20.2 \pm 6.5$ & $11.3 \pm 0.3$ & $<0.05$ \\
\hline $\begin{array}{c}0.6 \text { Hyperglycemia } \\
110 \mathrm{mg} / \mathrm{dl}\end{array}$ & 6 & 94 & $2.4 \dagger$ & $1.7 \pm 0.6$ & - & $0.9 \dagger$ & $0.5 \pm 0.03$ & - & $16.4 \dagger$ & $11.2 \pm 0.7$ & - \\
\hline
\end{tabular}

$\mathrm{P}$ value calculated based on log-transformed concentration of CRP. † Unstable estimates.

Table 5. Results of multiple linear regression analysis with concentration of log-transformed hsCRP as the dependent variable among patients.

\begin{tabular}{|c|c|c|c|}
\hline Independent variables & $\begin{array}{l}\text { Regression } \\
\text { coefficient }\end{array}$ & SE & $\mathrm{P}$ \\
\hline Age (years) & 0.104 & 0.129 & 0.003 \\
\hline $\begin{array}{l}\text { Sex (male vs. female) } \\
\text { (ref.) }\end{array}$ & -0.068 & 0.113 & 0.524 \\
\hline $\begin{array}{l}\text { Abdominal obesity } \\
\text { Yes vs. no (ref.) }\end{array}$ & 1.876 & 0.123 & 0.001 \\
\hline $\begin{array}{l}\text { Hypertriglyceridemia } \\
\text { Yes vs. no (ref.) }\end{array}$ & -0.061 & 0.132 & 0.670 \\
\hline $\begin{array}{c}\text { HDL < } 40 \\
\text { Yes vs. no (ref.) }\end{array}$ & 0.157 & 0.120 & 0.117 \\
\hline $\begin{array}{l}\text { High blood pressure } \\
\text { Yes vs. no (ref.) }\end{array}$ & 0.257 & 0.264 & 0.134 \\
\hline $\begin{array}{l}\text { Glucose_110 } \\
\text { Yes vs. no (ref.) }\end{array}$ & 0.038 & 0.253 & 0.139 \\
\hline
\end{tabular}

hypertriglyceridemia, $28 \%$ had a low concentration of HDL cholesterol, $18 \%$ had high blood pressure and $6 \%$ had a concentration of glucose $\geq 110 \mathrm{mg} / \mathrm{dL}$. Mean and geometric mean concentrations of hs CRP were higher among patients who had the metabolic syndrome (mean $3.8 \mathrm{mg} / \mathrm{L}$, geometric mean $1.9 \mathrm{mg} / \mathrm{L}$ ) than among those who did not (mean $1.3 \mathrm{mg} / \mathrm{L}$, geometric mean $0.4 \mathrm{mg} / \mathrm{L}$ ). Among patients with the metabolic syndrome, $35 \%$ had a concentration of hsCRP $>3.0 \mathrm{mg} / \mathrm{L}$, a concentration considered to place adults at high risk for cardiovascular disease. In comparison, $12.7 \%$ of adolescents without the syndrome had such a concentration of hsCRP $(\mathrm{P}<$ 0.05 ).

Table 5 shows results of multiple linear regression analysis with concentration of log-transformed CRP as the dependent variable among patients.

Of the five components of the metabolic syndrome, mean concentration of hs CRP was higher only among those with abdominal obesity. However, mean concen- trations of log-transformed hsCRP were higher among patients with abdominal obesity, hypertriglyceridemia, low HDL cholesterol, and high blood pressure compared with patients without those conditions. In multiple logistic regression analysis with age, sex, and all five components of the metabolic syndrome added as independent variables, only abdominal obesity was significantly and independently associated with log-transformed concentration of hsCRP. When the same five components were examined as continuous variables in another linear regression model, only waist circumference was significantly associated with concentrations of log-transformed hsCRP. Results from an analogous logistic regression model with dichotomized concentration of hsCRP as the dependent variable and the five components added as continuous independent variables yielded similar conclusions.

\section{DISCUSSION}

While there have been many previous studies relating CRP and cardiovascular risk factors, the association of CRP with subclinical cardiovascular complication has been examined primarily in adults, with few studies in obese children [13]. Traditional risk factors for cardiovascular disease include aging, hypertension, dyslipidemia, smoking, and diabetes. The contribution of lipid accumulation to atherosclerotic disease is well known, but laboratory and experimental evidence indicates that chronic inflammatory processes also play an important role in the development of atherosclerosis [14]. In order to prevent the incidence of cardiovascular events, it is important to weigh the influence of each risk factor on the cardiovascular system [15]. In this respect, we investigated the relationship of inflammatory markers with other risk factors. In our study, we found that height, 
weight and BMI were significantly higher in the obesity group than in the control group. Also, obese group had significantly higher hs-CRP, LDL-C, TG and Apo B and significantly lower HDLC than the control group. These findings were in concordance with Quijada et al. (2008) who stated that, Systolic, diastolic, and mean blood pressures (MBP), low-density lipoprotein cholesterol (LDL-C), Tg/HDL-C, total cholesterol/HDL-C, LDL-C/ HDL-C ratios, CRP, and leptin were significantly higher in the obese group [16]. Our study showed elevated serum hsCRP levels were positively associated with elevated BMI, confirming previous observations in both children [17] and adults [18]. The mechanisms underlying this association with BMI or obesity might be as follows; the adipose tissue is a source of cytokines such as tumor necrosis factor-a (TNF-a) and interleukin-6 (IL-6), and these cytokines stimulate the production of acute-phase proteins such as C-reactive protein in the liver. Not unexpectedly, then, we found that serum hsCRP levels were positively associated with BMI [19]. Our results are in agreement of Martos et al. (2009) who reported that $\mathrm{C}$-reactive protein (CRP) levels were significantly $(\mathrm{P}<0.001)$ higher in obese children than in controls. After 9 months of treatment, obese children with lowered BMI SD score (SDS-BMI) displayed a significant decrease CRP $(\mathrm{P}=.006)$, levels compared with obese children with stable SDS-BMI. In addition to BMI, hsCRP was also found to be closely correlated with SBP, DBP, and ApoE and TG concentrations. Previous studies reported that CRP levels are significantly positively correlated with TG, total ratio of serum cholesterol to serum HDL cholesterol, fibrinogen levels, heart rate, SBP, smoking, and white blood cell count and negatively correlated with HDL-C levels [20]. In this study, the prevalence of the metabolic syndrome was $24 \%$. Mean and geometric mean concentrations of hs CRP were significantly higher among patients who had the metabolic syndrome than among those who did not. Among patients with the metabolic syndrome, $35 \%$ had a concentration of hsCRP $>3.0 \mathrm{mg} / \mathrm{L}$, a concentration considered to place adults at high risk for cardiovascular disease. In comparison, $12.5 \%$ of adolescents without the syndrome had such a concentration of hsCRP $(\mathrm{P}<0.05)$. Soriano-Guillén et al. (2008) carried out his study on 115 obese children, $24 \%$ showed signs of metabolic syndrome. Those with metabolic syndrome presented higher levels of hs-CRP in comparison with the obese patients who did not show signs of metabolic syndrome After a multivariate analysis, the variables that appeared to influence the changes in (hsCRP)were BMI, triglycerides and HDL-cholesterol levels. From his study he concluded that (hsCRP) is a useful tool for early diagnosis of cardiovascular risk in obese children and teenagers
[21]. Current estimates are that approximately $25 \%$ of American adults, and an increasing percentage of children and adolescents, can be classified as having Metabolic Syndrome. The wide prevalence of metabolic Syndrome makes this condition a major contributor to cardiovascular risk [6]. Of the five components of the metabolic syndrome, mean concentration of (hsCRP) was higher only among those with abdominal obesity. However, mean concentrations of log-transformed hs CRP were higher among patients with abdominal obesity, hypertriglyceridemia, low HDL cholesterol, and high blood pressure compared with patients without those conditions. Weiss et al., 2004 agreed that log-transformed (hsCRP) levels were affected by abdominal obesity, hypertriglyceridemia, hypertension, HDL cholesterol, and high blood pressure [22]. The metabolic syndrome has generated a great deal of interest in recent years. Among adults and adolescents, components of the metabolic syndrome and the metabolic syndrome itself are associated with measures of inflammation, such as concentrations of (CRP). This low-grade inflammation, which has been associated with an increased risk for cardiovascular disease and diabetes may provide a mechanism for the increased risk of these conditions experienced by individuals who have the metabolic syndrome, however, abdominal obesity was the component that was responsible for much of the difference in concentrations of (hsCRP) [23]. The present study showed association between obesity and elevated concentrations of (hsCRP) in children and adolescents. In univariate analysis, concentrations of (hsCRP) have also been significantly associated with the other four components of the metabolic syndrome. Our results suggest that the presence of the metabolic syndrome and abdominal obesity among children and adolescents may be laying the foundation for the emergence of cardiovascular disease and diabetes later in life through early low-grade inflammation. Unfortunately, the sample size was inadequate to provide results separately for males and females. Similar results were obtained by Cizmecioglu et al. (2009) as they found that Waist circumference had the highest sensitivity and specificity for predicting MS in their patients [24]. We appreciate the comments of Dr. Kholeif regarding the utility of (CRP) measurement in stratifying cardiovascular disease (CVD) risk as it relates to our results of patients with the metabolic syndrome categorizing CRP into normal ( $11 \mathrm{mg} / \mathrm{l})$, borderline $(1-3 \mathrm{mg} / \mathrm{L})$, and high-risk ( $3 \mathrm{mg} / \mathrm{l})$ levels are appropriate for stratifying patient risk in combination with other risk factor [25]. While our data must be interpreted cautiously because they are of a cross-sectional nature, our findings are consistent with those of Ridker et al. showing metabolic syndrome patients with elevated (hsCRP) levels to 
have a less optimistic prognosis than those with normal (hsCRP) levels [26]. Although our study relied on a single measurement as provided by the National Health and Nutrition Examination Survey (NHANES) study and thus did not have duplicate measures over time, Dr. Kholeif reported that a single CRP measurement, given its intraindividual biological variability, is not suitable and that the use of multiple measures would establish the certainty of a given level [25]. Recently, the Centers for Disease Control (CDC)/American Heart Association (AHA) workshop on markers of inflammation and cardiovascular disease did recommend that the mean of only two measures taken 2 weeks apart could be averaged to provide a clinically useful value [27]. The studies above demonstrate that vascular risk prediction and the prediction of type 2 diabetes can be improved by knowledge of (hsCRP) levels, even among those with metabolic syndrome. Recent studies relating (hsCRP) to incident hypertension serve to reinforce the importance of blood pressure in the metabolic syndrome complex [28,29] Investigators have long hypothesized links between the metabolic derangements of insulin resistance syndrome/type 2 diabetes and the development and progression of atherosclerosis. A number of investigators have similarly concluded that IL-6 and CRP are associated with hyperglycemia, insulin resistance, and overt type 2 diabetes, and both are strong predictors of cardiovascular disease in apparently healthy people [30]. Although ultrasonography allows visualization of early subclinical stages of atherosclerosis in obese children, the measurement of the serum (hsCRP) level is simpler and cheaper than ultrasonography, is highly reproducible, and well correlates with carotid intima-media wall thickness (IMT) and brachial flow-mediated dilation in obese children (FMD). Thus, (hsCRP) would be a useful screening marker for evaluating and estimating the degree of atherosclerosis in children [31].

\section{CONCLUSIONS}

Our results suggest that the presence of the metabolic syndrome and abdominal obesity among children and adolescents may be laying the foundation for the emergence of cardiovascular disease later in life through early low grade inflammation. hsCRP is one of the inflammatory markers that can be easily estimated in these patients.

\section{RECOMMENDATION}

hsCRP can be used as a useful screening test for prediction of cardiovascular changes in Obese children and adolescents. More work is needed to establish whether intervention targeting "highrisk" metabolic syndrome patients, identified on the basis of elevated (hsCRP), effectively lowers CVD risk. To reduce adverse effects of inflammation that accompanies the metabolic syndrome, children should avoid excessive energy intake, limit sedentary behavior, and increase their energy expenditure. In contrast to other biomarkers that also reflect inflammation, (hsCRP) measurement is inexpensive, standardized, widely available, and has a decade-to-decade variation similar to that of cholesterol. Given the consistency of prognostic data for (hsCRP) and the practicality of its use in outpatient clinical settings, we believe the time has come for a careful consideration of adding (hsCRP) as a clinical criterion for metabolic syndrome and for the creation of an (hsCRP) modified coronary risk score useful for global risk prediction.

\section{REFERENCES}

[1] Chinn S. and Rona, R.J. (2008) Prevalence and trends in overweight and obesity in three cross sectional studies of British children, 1974-1994. British Medical Journal, 322(7277), 24-26.

[2] Bruno, G., Fornengo, P., Novelli, G., Panero, F., Perotto, M., Segre, O., et al. (2009) C-reactive protein and 5-year survival in type 2 diabetes: The casale monferrato study. Diabetes, 58(4), 926-933.

[3] Ridker, P.M. (2007) CRP and the prediction of cardiovascular events among those at intermediate risk. Journal of the American College of Cardiology, 49(21), 2129. 2138.

[4] Sinha, R., Fisch, G., Teague, B., Tamborlane, W.V., Banyas, B., Allen, K., et al. (2002) Prevalence of impaired glucose tolerance among children and adolescents with marked obesity. The New England Journal of Medicine 346, 802-810.

[5] David, M., Capuzzi, A., Jeffrey, S. and Freeman (2007) CRP and cardiovascular risk in the Metabolic Syndrome. Clinical Diabetes, 25(1), 16-22.

[6] Chen, J. (2009) Metabolic syndrome and salt sensitivity of blood pressure in non-diabetic people in China, a dietary intervention study. Lancet, 373(9666), 829-835.

[7] Steinberger, J., Daniels, S.R. and Eckel, R.H. (2009): Progress and challenges in metabolic syndrome in children and adolescents. Circulation, published online.

[8] Ford, E.S., Galuska, D.A., Gillespie, C., Will, J.C., Giles, W.H. and Dietz, W.H. (2001) CRP and body mass index in children. Journal of Pediatric Surgery, 138(4), 486-492.

[9] Shea, S., Aymong, E., Zybert, P., Shamoon, H., Tracy, R.P., Deckelbaum, R.J., et al. (2003) Obesity, fasting plasma insulin and CRP levels in healthy children. Obesity Research, 11(1), 95-103.

[10] Vikram, N.K., Misra, A., Dwivedi, M., Sharma, R., Pandey, R.M., Luthra, K., et al. (2003) Correlations of CRP levels with anthropometric profile. Atherosclerosis, 168(2), 305-313.

[11] Roberts, W.L., Moulton, L. and Law, T.C. (2001) Evaluation of nine automated (hsCRP) methods. Clinical Che- 
mistry, 47(5), 979-980.

[12] Goldstone, L.A. (1983) Statistical tests and concepts of significance. Understanding Medical Statistics, 1st Edtion, William Heinman Medical Books Ltd., London.

[13] Cook, D.G., Mendall, M.A., Whincup, P.H., Carey, I.M., Ballam, L., Morris, J.E., et al. (2000) C-reactive protein concentration in children: relationship to adiposity and other cardiovascular risk factors. Atherosclerosis, 149(1), 139-150.

[14] Ryu, S.Y., Lee, Y.S., Park, J., Kang, M.G. and Kim. K.S. (2005) Relations of plasma (hsCRP) to various cardiovascular risk factors. Journal of Korean Medical Science, 20(3), 379-383.

[15] Caballero, A.E., Bousquet-Santos, K., Robles-Osorio, K., Montagnani, V., Soodini, G., Porramatikul, S., et al. (2008) Overweight latino children and adolescents have marked endothelial dysfunction and subclinical vascular inflammation. Diabetes Care, 31(3), 576-582.

[16] Quijada, Z., Paoli, M., Zerpa, Y., Camacho, N., Cichetti, R., Villarroel, V., et al. (2008) The triglyceride/HDLcholesterol ratio as a marker of cardiovascular risk in obese children; association with traditional and emergent risk factors. Pediatric Diabetes, 9(5), 464-471.

[17] Lambert, M., Delvin, E.E., Paradis, G., O’Loughlin, J., Hanley, J. and Levy, E. (2004) CRP and metabolic syndrome children and adolescents. Clinical Chemistry, 50, 1762-1768.

[18] Ruckerl, R., Peters, A., Khuseyinova, T., Andreani, M., Koenig, W., Meisinger, C., et al. (2009) Determinants of the acute-phase protein c-reactive protein in myocardial infarction survivors. Clinical Chemistry, 55(2), 322-335.

[19] Hiura, M., Kikuchi, T., Nagasaki, K. and Uchiyama, M. (2003) Elevation of serum CRP levels is associated with obesity in boys. Hypertension Research, 26(7), 541-546.

[20] Martos, R., Valle, M., Morales, R.M., Cañete, R., Gascón, F. and Urbano, M.M. (2009) Changes in body mass index are associated with changes in inflammatory and endothelial dysfunction biomarkers in obese prepubertal children after 9 months of body mass index SD score loss. Metabolism.

[21] Soriano-Guillén, L., Hernández-García, B., Pita, J., Domínguez-Garrido, N., Del Río-Camacho, G., et al. (2008) HsCRP is a good marker of cardiovascular risk in obese children and adolescents. European Journal of Endocri- nology, 159(1), R1-R4.

[22] Weiss, R., Dziura, J., Burgert, T.S., Tamborlane, W.V., Taksali, S.E., Yeckel, C.W., et al. (2004) Obesity and the metabolic syndrome in children and adolescents. The New England Journal of Medicine, 350(23), 2362-2374.

[23] Danesh, J., Wheeler, J.G., Hirschfield, G.M., Eda, S., Eiriksdottir, G., Rumley, A., et al. (2004) CRP and other circulating markers of inflammation in the prediction of coronary heart disease. The New England Journal of Medicine, 350(14), 1387-1397.

[24] Cizmecioglu, F.M., Etiler, N., Ergen, A., Gormus, U., Keser, A., Hekim, N., et al. (2009) Association of adiponectin, resistin and hscrp level with the metabolic syndrome in childhood and adolescence. Experimental and Clinical Endocrinology \& Diabetes, 117(10), 622-627.

[25] Kholeif, M.A. (2005) CRP for cardiovascular risk assess. Diabetes Care, 28(7), 1833-1834.

[26] Ridker, P.M., Wilson, P.W.F. and Grundy, S.M. (2004) Should CRP be added to metabolic syndrome and to assessment of global cardiovascular risk? Circulation, 109 (23), 2818-2825.

[27] Myers, G.L., Rifai, N., Tracy, R.P., Roberts, W.L., Alexander, R.W., Biasucci, L.M., et al. (2005) The Centers for Disease Control, the American Heart association: CDC/ AHA workshop on markers of inflammation and cardiovascular disease. Circulation, 110(6), 22-26.

[28] Reaven, P.D., Traustadottir, T., Brennan, J. and Nader, P.R. (2005) Cardiovascular risk factors associated with insulin resistance in children persist into late adolescence. Diabetes Care, 28(1), 148-150.

[29] Kriketos, A.D., Greenfield, J.R., Peake, P.W., Furler, S. M., Denyer, G.S., Charlesworth, J.A. and Campbell, V., et al. (2004) Inflammation, insulin resistance, and adiposity: A study of first-degree relatives of type 2 diabetes. Diabetes Care, 27(8), 2033-2040.

[30] Mattsson, N., Rönnemaa, T., Juonala, M., Viikari, J.S. and Raitakari, O.T. (2008) Childhood predictors of the metabolic syndrome in adulthood. Annals of Medicine, 40(7), 542-552.

[31] Roh, E.J., Lim, J.W., Kyoung, O.K. and Cheon, E.J. (2007) A useful predictor of early atherosclerosis in obese children: hsCRP. Journal of Korean Medical Science, 22(2), 192-197. 